\title{
A Novel Anatomical Self-locking Plate Fixation for Both-column Acetabular Fractures: - Finite Element Analysis
}

\section{Ming Li ( $\sim$ angells6@163.com)}

Department of Traumatic Orthopaedics, the Medical College of Ningbo University Affiliated Ningbo N0.6 Hospital, 1059 Zhongshan East Road, Ningbo 315040

\section{Ding Xu}

Department of Traumatic Orthopaedics, the Medical College of Ningbo University Affiliated Ningbo N0.6 Hospital, 1059 Zhongshan East Road, Ningbo 315040

\section{Yong Zhang}

Department of Traumatic Orthopaedics, the Medical College of Ningbo University Affiliated Ningbo N0.6 Hospital, 1059 Zhongshan East Road, Ningbo 315040

\section{Bo Chen}

Department of Orthopaedics, the Shanghai Jiaotong University School of Medicine Affiliated Shanghai Ruijin Hospital, Shanghai 200025

\section{Haiyang Li}

Department of Traumatic Orthopaedics, the Medical College of Ningbo University Affiliated Ningbo N0.6 Hospital, 1059 Zhongshan East Road, Ningbo 315040

\section{Shuaiyi Wang}

Department of Traumatic Orthopaedics, the Medical College of Ningbo University Affiliated Ningbo N0.6 Hospital, 1059 Zhongshan East Road, Ningbo 315040

\section{Research Article}

Keywords: Both column acetabular fractures, Internal fixation, Three dimensional finite element, Biomechanics

Posted Date: March 15th, 2021

DOI: https://doi.org/10.21203/rs.3.rs-288024/v1

License: (c) (i) This work is licensed under a Creative Commons Attribution 4.0 International License. Read Full License 


\section{A Novel Anatomical Self-locking Plate Fixation for Both-column Acetabular}

\section{Fractures: - Finite Element Analysis}

Ming $\mathrm{Li}^{1,{ }^{*}, \#}$, Ding $\mathrm{Xu}^{1, \#}$, Yong Zhang ${ }^{1,}$, Bo Chen ${ }^{2}$, Haiyang $\mathrm{Li}^{1}$, Shuaiyi Wang ${ }^{1}$

*Correspondence :angells6@163.com

\#Co-first author: Ding Xu

1 Department of Traumatic Orthopaedics, the Medical College of Ningbo University Affiliated Ningbo NO.6 Hospital, 1059 Zhongshan East Road, Ningbo 315040, China.

2 Department of Orthopaedics, the Shanghai Jiaotong University School of Medicine Affiliated Shanghai Ruijin Hospital, Shanghai 200025, China

\section{Abstract}

Background: Both-column acetabular fractures often require multiple plates for fixation, and the risk of internal implant failure is high. The author designed a posterior anatomic self-locking plate (PASP) to avoid the shortcomings. The stability of PASP was compared with two popular reconstruction plate fixation methods, and the influence of sitting, turning right and left on implants were explored.

Methods: PASP, double reconstruction plate (DRP), and cross reconstruction plate (CRP) were assembled on the finite element model of both-column fractures of the left acetabulum. A load of $600 \mathrm{~N}$ and a torque of $8 \mathrm{~N} \bullet \mathrm{m}$ were loaded on the S1 vertebral body to detect stress and displacement changes when sitting, turning right and left.

Results: The peak stress and displacement of three types of fixation methods on the left both-column fractures under three kinds of movements were CRP > DRP > PASP. PASP has the minimal value when turning left. The maximum peak of stress and displacement of PASP are $313.5 \mathrm{MPa}$ and $1.15 \mathrm{~mm}$ respectively when turning right.

Conclusion: PASP can provide higher stability than two reconstruction plates for both-column acetabular fractures. The rational movement after posterior DRP and PASP fixation for acetabular fracture is to turn to the ipsilateral side, which can avoid implant failure.

Keywords: Both-column acetabular fractures; Internal fixation; Three-dimensional finite element; Biomechanics

\section{Background}

Both-column acetabular fractures are high energy injuries such as falls from heights, car accidents, and massive object crashes. It was the most severe damage to the stability of the hip joint, and multiple reconstruction plates are required for fixation. The operation complications include unstable or ineffective fixation of fracture fragments, screw invasion into the joint. Postoperative complications include screw loosening, slippage or rupture, reduction loss etc. This will lead to severe traumatic arthritis, and the end result is total hip replacement (THR) [1,2].

Double reconstruction plate (DRP) is the most popular method for posterior acetabular fixation $[3,4]$. When there are more than 3 comminuted fragments of the posterior wall and 
column of the acetabulum, 2 4 plates are often needed for fixation [5,6]. Individualized three-dimensional (3D) printing plates are not yet popular [7]. The most widely used reconstruction plate has good flexibility and easy to be manual remodelled according to the morphological characteristics of the acetabulum surface of the patient during operation. However, the disadvantages are that manual remodelling will leave scratches on the plate, increase the internal stress concentration in the plate and the risk of implant failure. It is difficult to ensure that the remodelled plate fits the bone, and screw insertion is easy to invade the joint. In addition, intraoperative plate remodelling will delay the operation time, increase wound bleeding and the chance of infection, prolong the wound exposure time. If the plate is poorly remodelled, or there is shear force between two overlapped plates, it will lead to stress shielding, implant loosening, reduction loss, and increase the risk of nonunion or malunion[1,2]. Patients cannot be guaranteed early functional movements to restore hip joint function. Currently there is a lack of effective internal fixation devices for osteoporosis patients $[8,9]$. Based on the above background, the author designed the posterior anatomic self-locking plate (PASP) (patent number: ZL201310371875.8) on the basis of acetabular anatomy, biomechanics, and ergonomics. The radian and torsion angle of PASP matches the morphological characteristics of the posterior acetabulum, and it includes six functional fixation zones. Even if the fracture of the posterior acetabulum is severely comminuted, the bone can be effectively fixed.

The purpose of this study is, firstly, to compare the stability of PASP, DRP and cross reconstruction plate (CRP) [10], and to determine the most stable fixation mode. The second is to explore the influence of sitting, turning right and left movements on three types of implants, and to identify the movement that has the minimal influence on implant.

\section{Methods}

\section{Ethical review}

The Ethics Committee approved this study of the Medical College of Ningbo University affiliated Ningbo NO.6 Hospital and involved the examination of an adult volunteer with a written informed consent before the study began (sex: male, age: 45 , height: $165 \mathrm{~cm}$, weight: $61 \mathrm{~kg}$ ) by enhanced computed tomography (CT) to obtain raw imaging data of a normal pelvis. All methods were carried out in accordance with relevant guidelines and regulations. The datasets used and analysed during the current study are available from the corresponding 
author on reasonable request.

\section{The indications of PASP}

PASP can be used in OTA classification type 62A1/A2, 62B1/B2/B3, 62C1/C2[11]. The details are as follows, (1) Comminuted fractures of the posterior wall and posterior column of acetabulum. (2) Posterior acetabulum combined with weight-bearing dome, including compressed and collapsed fracture. (3) Fractures of the posterior acetabulum combined with medial quadrilateral region. (4) It is suitable for the fixation of the posterior and medial bone grafts during THR.

\section{D modeling}

The scanning parameters of the CT machine (SOMATOM Definition AS128, Siemens AG): $120 \mathrm{kV}, 250 \mathrm{~mA}$, slice $0.6 \mathrm{~mm}$, pitch $0.55 \mathrm{~mm}$, and the scanned image is saved in DICOM format. DICOM data was imported into Mimics 12.1 software (Materialise, Leuven, Belgium) and Geomagic Studio V12.0 (Raindrop Company, USA) to establish the pelvic model (Fig.1a). The computer-aided design (CAD) modeling of titanium alloy implants were completed in Solidworks software (Solidworks/UGS V2010, Dassault Systemes SA, France). The reconstruction plate parameters: $2.8 \mathrm{~mm}$ (thickness), $10.0 \mathrm{~mm}$ (width) and $3.5 \mathrm{~mm}$ (solid screw diameter). The average plate thickness and a screw diameter of the PASP are the same as the reconstruction plate to ensure the homogeneousness of comparison (Fig.2b-c).

\section{Finite element (FE) modeling and material properties}

The ilium and sacrum are divided into the cortical and cancellous bone, and the bones are all C3D4 units (4 nodes and 4 tetrahedral unit) [12]. The ligaments were used by Spring2 units [12-14]. According to the characteristics of anatomical structure of the pelvis, seven ligaments related to pelvic stability were established: sacroiliac ligament, sacrospinous ligament (SSL), sacrotuberous ligament (STL), iliolumbar ligament, inguinal ligament, superior pubic ligament, arcuate pubic ligament (Fig.1b). According to Rice's [15] , Carter's [16], and Phillips research [13], the material properties of the seven ligaments are shown in Table 1.

\section{Table 1 Material properties of the seven pelvic ligaments}

The six directions of displacement are as follows: the $\mathrm{X}$ direction is the sagittal plane, the $\mathrm{Y}$ direction is perpendicular to the sagittal plane and turn to the left, the $\mathrm{Z}$ direction is perpendicular to the coronal plane and forward. 


\section{Boundary conditions and validation of model}

The reference point established above the S1 vertebral body is distribution coupling with all the unit nodes on the upper edge of the S1 [17]. In order to simulate the human sitting posture and the movement states of turning right and left, six directions of bilateral ischial tuberosity in $\mathrm{XYZ}$ triaxis were constrained. A load of $600 \mathrm{~N}$ (equivalent to $60 \mathrm{~kg}$ body weight) was applied to the reference point along the direction of gravity, and a torque of $8 \mathrm{~N} \cdot \mathrm{m}$ was applied to the right and left directions respectively for a period of $1 \mathrm{~S}$. Model verification was performed in software Abaqus 6.9 (Dassault Systemes SIMULIA Corp., USA). It showed that the stress is mainly distributed through the sacrum, sacroiliac joint, arcuate line, posterior column and ischial tuberosity (Fig. 1c) with the normal sitting position. The displacement distribution was similar to waves (Fig. 1d) which was consistent with previous literature reports [18-22]. From blue to red, the distribution nephogram values gradually increases.

Fig. 1 a Pelvic solid model, b Finite element model of the pelvis and its seven ligaments, c/d Model validation, stress (c) and displacement distribution (d) of the sitting posture under a vertical load of $600 \mathrm{~N}$

\section{Fracture model and internal implant assembly}

According to the morphological characteristics described by OTA classification type 62C1 [11], the model of left both-column acetabular fracture was established (Fig. 2a). There were three fracture fragments in the anterior and posterior columns respectively, and acetabular joint was not connected to the axial sacrum. The SSL and STL on the left were missing. According to the requirements of clinical surgery, three types of implants were assembled on this model: PASP (Fig. 2b), DRP (Fig. 2c), and anterior CRP (Fig. 2d). Each type of implant was assembled with 8 screws of $3.5 \mathrm{~mm}$ diameter for homogeneousness comparison. The nine points of stress and displacement measurement are as follows: 1. right iliac fossa, 2. left iliac fossa, 3. right sacral ala, 4. left sacral ala, 5. right arcuate line, 6. left arcuate line, 7. right pubic tubercle, 8 . left pubic tubercle, 9. sacral promontory.

Fig. 2 a The finite element model of the left both-column acetabular fracture with 9 measurement points of stress and displacement. The SSL and STL are missing on the left. b/ $c / d$ Assembly diagram of PASP, DRP, CRP

Von Mises stress (VMS) patterns were used for stress analysis of various parts of the implants. The peak stress of implant indicates the risk of metal fatigue rupture is likely to occur. The peak displacement represents the largest movement distance of the plate or screw, which are 
prone to implant loosening.

\section{Results}

The numbers of total nodes were 103178, 135895 and 121593 in PASP, DRP and CRP models respectively and total number of elements were329974, 522867 and 440046 for PASP, DRP and CRP respectively. The load is positively correlated with stress and displacement, which is consistent with the relevant literature reports [18] . The stress and displacement of three types of implants under three kinds of loads are analyzed as follows.

Vertical Load

Fig .3 Nephogram of stress (a/b/c) and displacement distribution (d/e/f) under vertical loading in PASP

Fig . 4 Nephogram of stress (a/b/c) and displacement distribution (d/e/f) under vertical loading in DRP

Fig .5 Nephogram of stress (a/b/c) and displacement distribution(d/e/f) under vertical loading in CRP

Under vertical load, the stress peaks of PASP (Fig. 3), DRP (Fig. 4) and CRP (Fig. 5) are 262.1 MPa, 300.6 MPa and 439.2 MPa respectively, and the displacement peaks are $0.87 \mathrm{~mm}$, $0.96 \mathrm{~mm}$ and $2.24 \mathrm{~mm}$ respectively. The order of stress and displacement peak values of three types of implants is as follows: CRP $>$ DRP $>$ PASP.

Turning Right and Left

Fig . 6 Stress and displacement distribution nephogram of turning right. a/b PASP, c/d DRP, e/f CRP

Fig . 7 Stress and displacement distribution nephogram of turning left. a/b PASP, c/d DRP, e/f CRP Under turning right (Fig. 6) and left (Fig. 7) load, the stress peaks of PASP, DRP and CRP are 313.5/218.3 MPa 383.0/234.0 Mpa, and 442.4/436.0MPa respectively. The displacement peaks are 1.15/0.62 $\mathrm{mm}, 1.26 / 0.67 \mathrm{~mm}$ and 2.10/2.41 $\mathrm{mm}$ respectively. The order of stress and displacement peak values under turning right and left load is as follows: $\mathrm{CRP}>\mathrm{DRP}>$ PASP. The comparison results consistent with the vertical load.

Fig. 8 The peak stress (a) and displacement (b) of three types of impants for both-column acetabular fractures 
It can be seen from Fig. 8, that the order of the stress and displacement peak values of three types of implants under three kinds of loads is CRP > DRP > PASP. When turning left, the peak values of PASP and DRP were less than that of turning right. The stress impact and displacement on CRP is basically the same under three loading conditions.

\section{Discussion}

The peak displacement and stress of PASP are the minimal among three types of internal fixation methods (Fig. 3/6-8), which shows that PASP has the best stability and has the least prone to implant failure. Its clinical importance is that PASP can be used to replace CRP or DRP in operation to provide higher stability.

In the phase of turning left, the peak stress and displacement on DRP and PASP decreased with the increase of time (Fig. 8), which indicated that this movement can maintain the stability of internal fixation and implant was not easy to loose or rupture. The clinical significance is that it is safe to turn left after operation for the both-column fractures of left acetabular with posterior plate fixation. It can be conjectured that ipsilateral rotation of the posterior plate fixation can reduce the implant failure. Under three kinds of loading conditions (Fig. 5-8), the changes of stress and displacement of CRP are minimal, indicating that the effects on implants are basically the same. Its clinical significance is that it is safe to turn to both sides after CRP fixation of acetabular fracture.

To the best knowledge of the authors, no biomechanical research data of postoperative movement after internal fixation of both-column acetabular fractures is available in the literature. There are no finite element studies of pelvis and its adjacent abdominal and spinal muscles [13]. Due to the complex morphology of both-column acetabular fracture, it is difficult to establish a biomechanical finite element model. The current biomechanical finite element study of acetabular internal fixation has the following problems: 1. Inaccurate description of fracture morphology. 2. The width and thickness of the plate, the diameter and length of screw are not defined, resulting in low repeatability of experiment [18,23-24]. Biomechanical studies have shown that the stress on the weight-bearing area of acetabular dome is the most concentrated, which requires anatomical reduction and effective fixation[21,22]. If the implant cannot effectively fix the displaced articular fragments, postoperative movements will result in reduction loss, implant loosening or rupture. The articular surface is not smooth and the friction between the femoral head and the acetabular 
cartilage surface will cause traumatic arthritis which requires THR.

PASP provides a new posterior fixation technique for acetabular fractures, which is designed according to the principles of tension band, pressure band, load sharing [25]. The shape of the plate is basically consistent with the distribution area of the pression and tension trabeculae of the acetabulum, which can reasonably disperse the compressive and tensile stress of the hip joint and conforms to the mechanical conduction path of the acetabulum bone. PASP includes six functional fixation regions: ilium, weight-bearing dome, posterior wall, posterior column, medial quadrilateral, and ischial branch. The locking screw on the plate effectively fixed the dissociated fracture fragments, maintained anatomical reduction to prevent bone loss, and prevented the displacement of comminuted fragments during postoperative movements, and is also suitable for osteoporosis patients. All locking screws do not invade the hip joint to ensure safety. The vacant screw hole is used as a vascular growth channel, that is, the soft tissue growth channel, which is beneficial to the blood supply reconstruction of the fracture site. It bears the movement load of hip joint and helps the fracture to heal stably in the most beneficial way for the patient, which meets the requirements of enhanced recovery after surgery (ERAS). PASP is helpful to complete the operation safely and effectively.

The limitations of this study is simulated the bone and ligaments damage, and the stability offered by the surrounding muscles was ignored. But, this technical limitation affected all the groups equally and it didn't question the validity of our findings. However, further studies are needed to explore the walking conditions in future research work.

\section{Conclusions}

The use of PASP instead of two reconstruction plates can provide higher stability for both-column acetabular fractures. The rational postoperative movement after posterior plate fixation of acetabular fracture is to turn to the ipsilateral side, which can reduce the implant failures. This study evaluated a new technique of PASP fixation, which provided an alternative option for the clinical internal fixation of acetabular fracture and the safe postoperative movements.

\section{Abbreviations}

THR: Total hip replacement; PASP: Posterior anatomic self-locking plate; DRP: Double reconstruction plate; CRP: Cross reconstruction plate; 3D: Three-dimensional; CT: Computed tomography; OTA: Orthopaedic Trauma Association; CAD: Computer aided design; SSL: Sacrospinous ligament; STL: Sacrotuberous ligament; 
VMS: Von Mises stress; ERAS: Enhanced recovery after surgery

\section{Acknowledgements}

Not applicable.

\section{Ethics approval and consent to participate}

This study has been approved by the Independent Ethics Committee of the Evaluation Committee of Ningbo NO.6 Hospital, and the written informed consent of volunteers has been obtained.

\section{Funding}

There is no source of funding related to this work.

\section{Availability of data and materials}

The datasets used and analysed during the current study are available from the corresponding author on reasonable request.

\section{Authors' contributions}

ML: Drafted the article and conceived of the study. DX: Manuscript preparation. YZ: Participated in the design of the study. BC: Data analysis and interpretation, finite element analysis. LHY: Data acquisition. WSY: Data acquisition. All authors reviewed and accepted the final manuscript.

\section{Consent for publication}

Not applicable.

\section{Competing interests}

The authors declare that they have no competing interests.

\section{Author details}

${ }^{1}$ Department of Traumatic Orthopaedics, the Medical College of Ningbo University Affiliated Ningbo NO.6 Hospital, 1059 Zhongshan East Road, Ningbo 315040, China. ${ }^{2}$ Department of Orthopaedics, the Shanghai Jiaotong University School of Medicine Affiliated Shanghai Ruijin Hospital, Shanghai 200025, China Correspondence: angells6@163.com, Co-first author: Ding Xu

\section{Publisher's Note}

Springer Nature remains neutral with regard to jurisdictional claims in published maps and institutional affiliations.

\section{References}

1. Lichte P, Sellei RM, Kobbe P, Dombroski DG, Gänsslen A, Pape HC.Predictors of poor outcome after both column acetabular fractures: a 30-year retrospective cohort study .Patient Saf Surg. 2013;7(1):9.

2. Ziran N, Soles GLS, Matta JM.Outcomes after surgical treatment of acetabular fractures: a review.Patient Saf Surg. 2019;13:16.

3. Li H, Yang $H$, Wang $D$, et al. Fractures of the posterior wall of the acetabulum: treatment using internal fixation of two parallel reconstruction plates. Injury. 2014;45(4):709-14.

4. Tosounidis TH, Giannoudis PV. What is new in acetabular fracture fixation? Injury. 2015; 46(11):2089-92.

5. Bhandari M, Matta J, Ferguson T, et al. Predictors of clinical and radiological outcome in patients with fractures of the acetabulum and concomitant posterior dislocation of the hip. J Bone Joint Surg Br. 2006;88 (12):1618-24.

6. Pierannunzii L, Fischer F, Tagliabue L, Calori GM, d'Imporzano M. Acetabular Both-Column Fractures: Essentials of Operative Management. Injury. 2010;41 (11), 1145-9.

7. Maini L, Sharma A, Jha S, Sharma A, Tiwari A. Three-dimensional printing and patient-specific pre-contoured plate: future of acetabulum fracture fixation? Eur J Trauma Emerg Surg. 2018;44(2):215-224.

8. Butterwick D, Papp S, Gofton W, Liew A, Beaulé PE. Acetabular fractures in the elderly: evaluation and management.J Bone Joint Surg Am. 2015;97(9):758-68.

9. Capone A, Peri M, Mastio M.Surgical treatment of acetabular fractures in the elderly: a systematic 
review of the results.EFORT Open Rev. 2017;2(4):97-103.

10. Tosounidis TH, Gudipati S, Panteli M, Kanakaris NK, Giannoudis PV. The use of buttress plates in the management of acetabular fractures with quadrilateral plate involvement: is it still a valid option? Int Orthop. 2015;39(11):2219-26.

11. Meinberg EG, Agel J, Roberts CS, Karam MD, Kellam JF. Fracture and Dislocation Classification Compendium-2018. J Orthop Trauma. 2018. Suppl 1:S1-S170.

12. Wang B, Sun P, Yao H, Tu J, Xie X, Ouyang J, Shen J. Modular hemipelvic endoprosthesis with a sacral hook: a finite element study. J Orthop Surg Res. 2019;14(1):309.

13. Phillips AT, Pankaj P, Howie CR, Usmani AS. Simpson AH. Finite element modelling of the pelvis: inclusion of muscular and ligamentous boundary conditions. Med Eng Phys. 2007;29(7):739-48.

14. Hammer N, Steinke H, Lingslebe U, Bechmann I, Josten C, Slowik V, Böhme J. Ligamentous Influence in Pelvic Load Distribution. Spine J. 2013;13 (10): 1321-30.

15. Rice JC, Cowin SC, Bowman JA. On the dependence of the elasticity and strength of cancellous bone on apparent density. J Biomech. 1988;21(2):155-68.

16. Carter DR, Hayes WC. The compressive behavior of bone as a two-phase porous structure. J Bone Joint Surg Am. 1977;59(7):954-62.

17. Hao Z, Wan C, Gao X, Ji T. The effect of boundary condition on the biomechanics of a human pelvic joint under an axial compressive load: a three-dimensional finite element model. J Biomech Eng. 2011;133(10),1-9.

18. Lei J, Dong P, Li Z, Zhu F, Wang Z, Cai X. Biomechanical analysis of the fixation systems for anterior column and posterior hemi-transverse acetabular fractures. Acta Orthop Traumatol Turc. 2017;51(3):248-53.

19. Hammer N, Klima S. In-silico pelvis and sacroiliac joint motion-A review on published research using numerical analyses. Clin Biomech (Bristol, Avon). 2019;61:95-104.

20. Hu P, Wu T, Wang HZ, Qi XZ, Yao J, Cheng XD, Chen W, Zhang YZ. Influence of Different Boundary Conditions in Finite Element Analysis on Pelvic Biomechanical Load Transmission. Orthop Surg. 2017;9(1):115-22.

21. Levine RG, Renard R, Behrens FF, Tornetta P. Biomechanical consequences of secondary congruence after both-column acetabular fracture. J Orthop Trauma. 2002;16(2):87-91.

22. Watson PJ, Dostanpor A, Fagan MJ, Dobson CA. The effect of boundary constraints on finite element modelling of the human pelvis. Med Eng Phys. 2017;43:48-57.

23. Yildirim AO, Alemdaroglu KB, Yuksel HY, Öken ÖF, Ucaner A. Finite element analysis of the stability of transverse acetabular fractures in standing and sitting positions by different fixation options. Injury. 2015;46 Suppl 2:S29-35.

24. Bodzay T, Sztrinkai G, Kocsis A, Kozma B, Gál T, Váradi K. Comparison of different fixation methods of bicolumnar acetabular fractures. Eklem Hastalik Cerrahisi. 2018;29(1):2-7.

25. Stoffel K, Klaue K, Perren SM.Functional load of plates in fracture fixation in vivo and its correlate in bone healing. Injury. 2000;31(Suppl 2):S-37-50. 
Figures

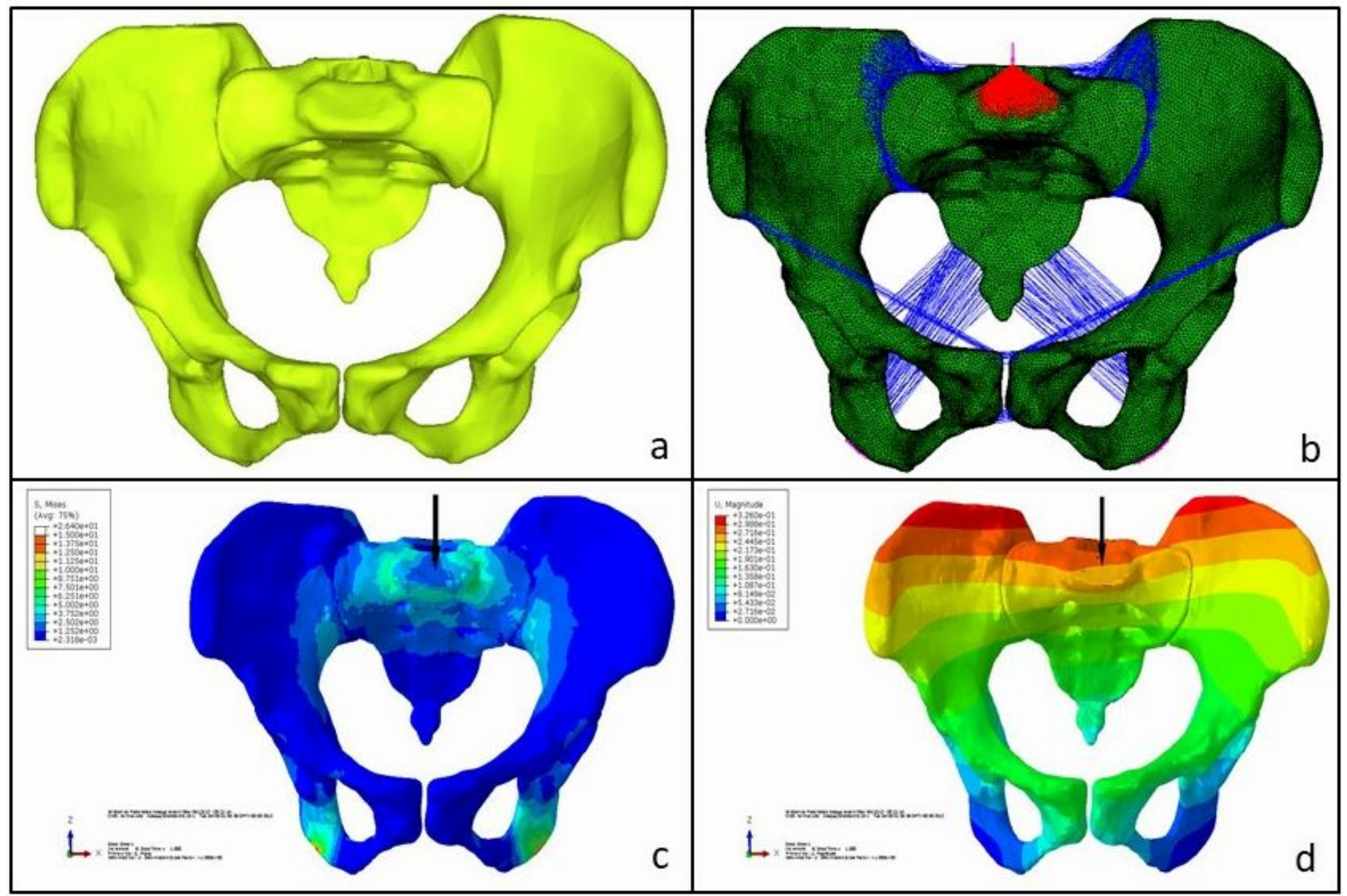

Figure 1

a Pelvic solid model, b Finite element model of the pelvis and its seven ligaments, c/d Model validation, stress (c) and displacement distribution d) of the $s$ itting posture under a vertical load of $600 \mathrm{~N}$ 

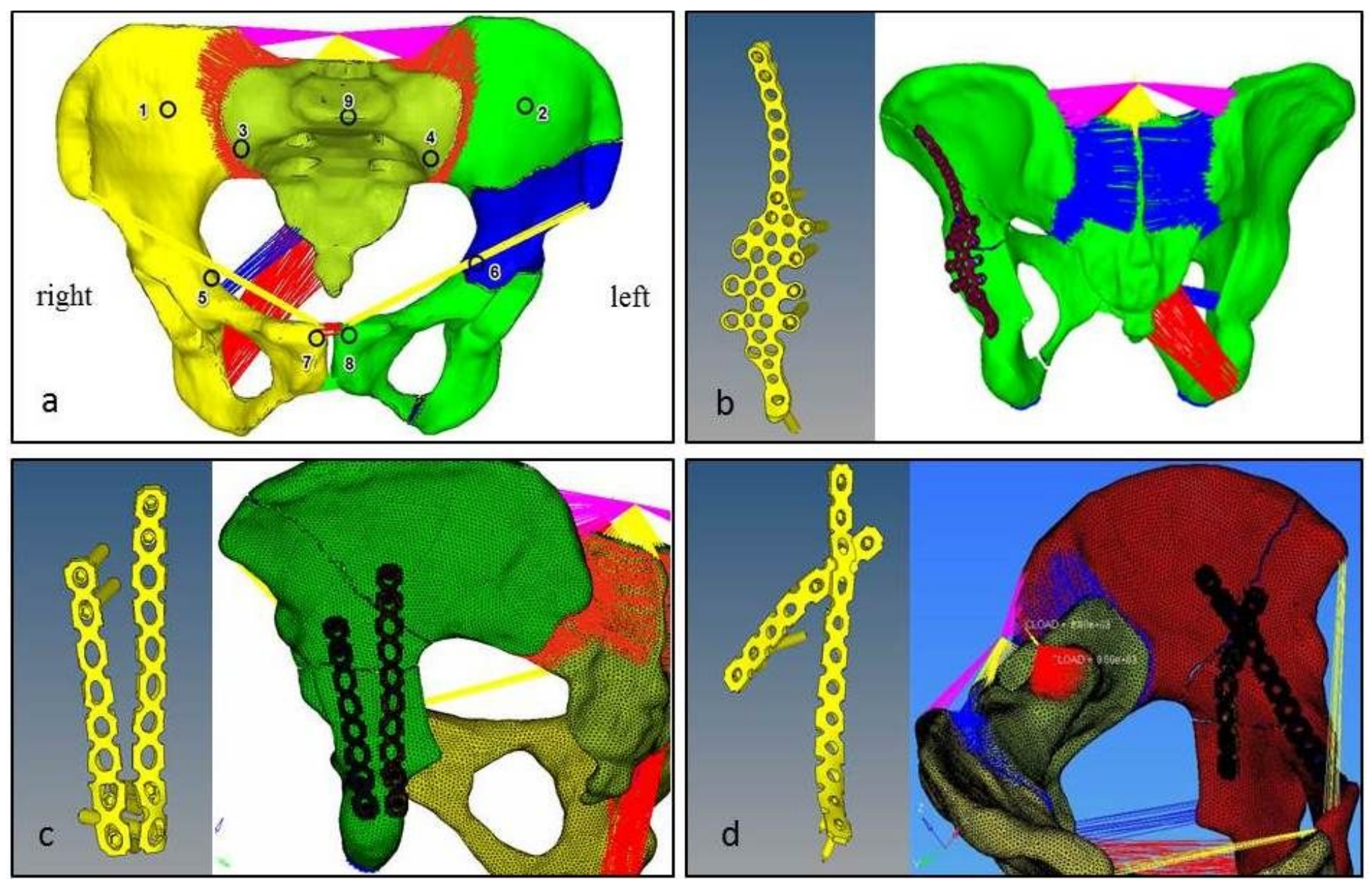

Figure 2

a The finite element model of the left both column acetabular fracture with 9 measurement points of stress and displacement. The SSL and STL are missing on the left . b c d Assembly diagram of PASP, DRP, CRP

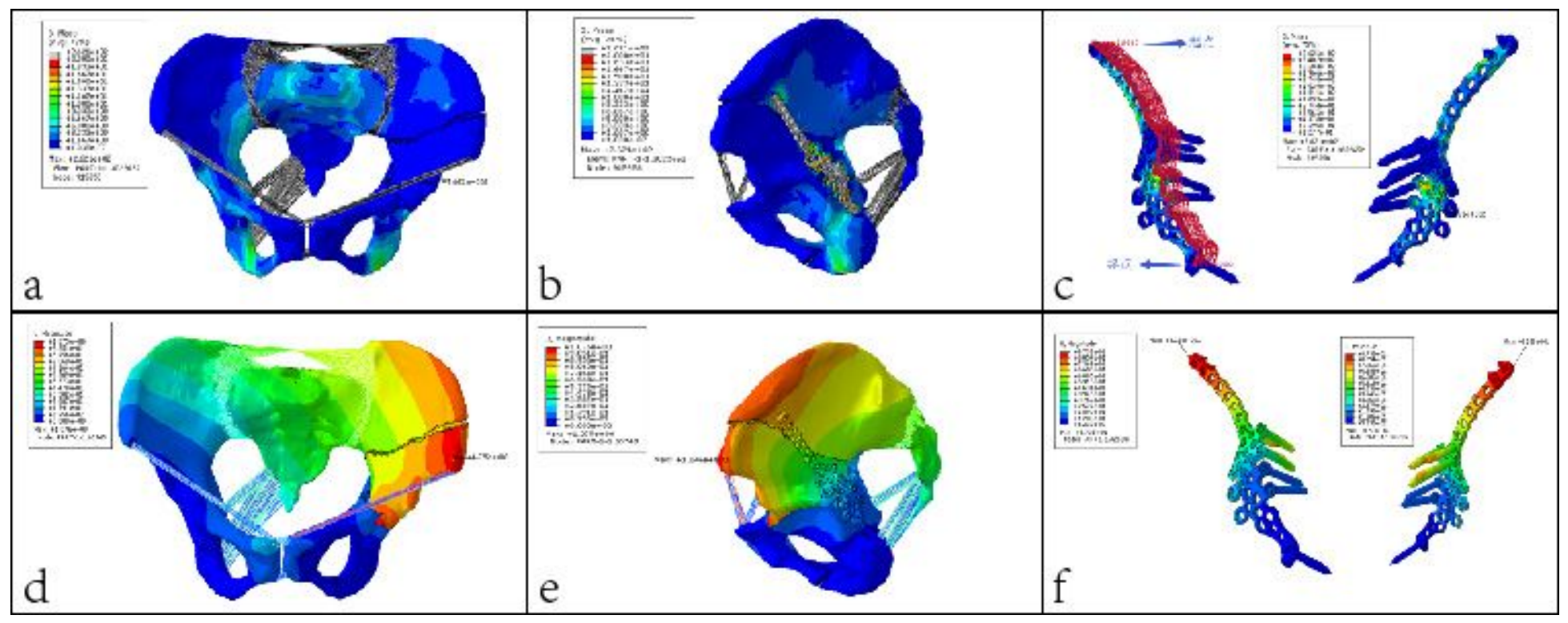

Figure 3 
Nephogram of stress $\mathrm{a} / \mathrm{b} / \mathrm{c}$ ) and displacement distribution $\mathrm{d} / \mathrm{e} / \mathrm{f}$ ) under vertical loading in PASP

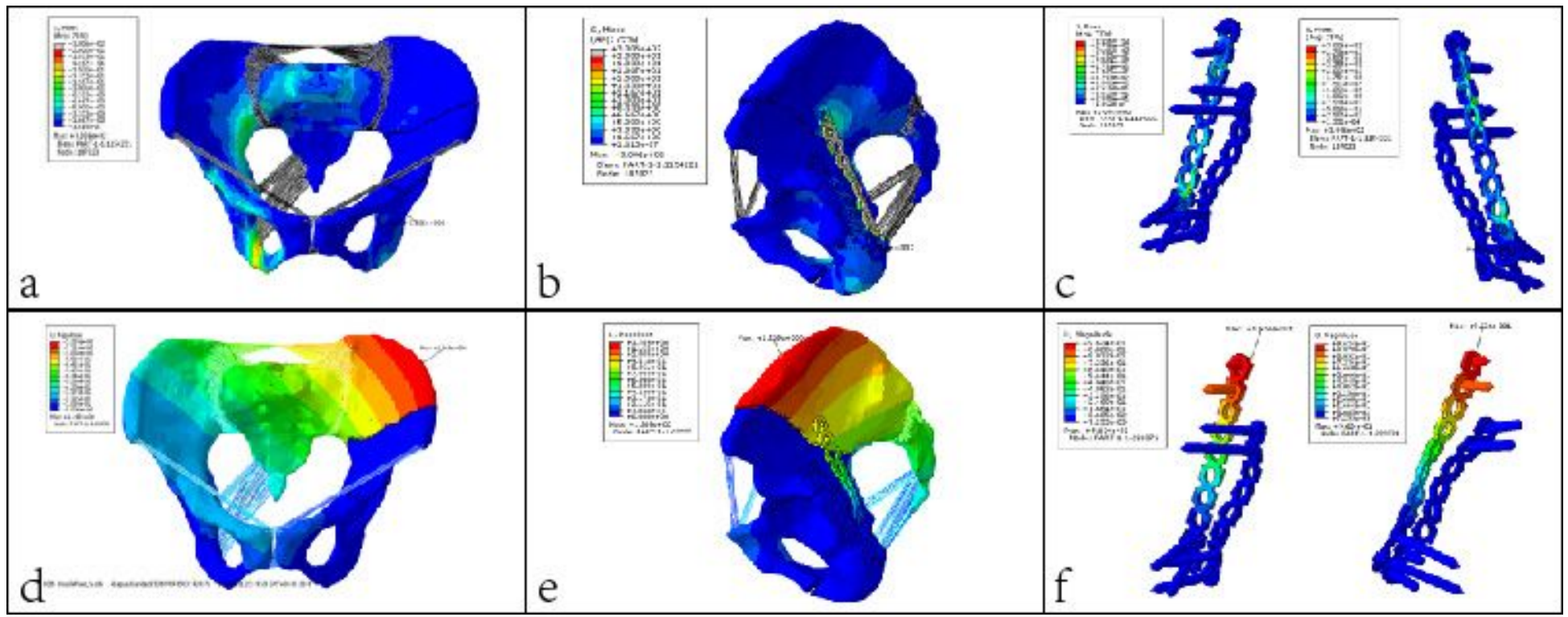

Figure 4

Nephogram of stress a/b/c ) and displacement distribution d/e/f under vertical loading in DRP

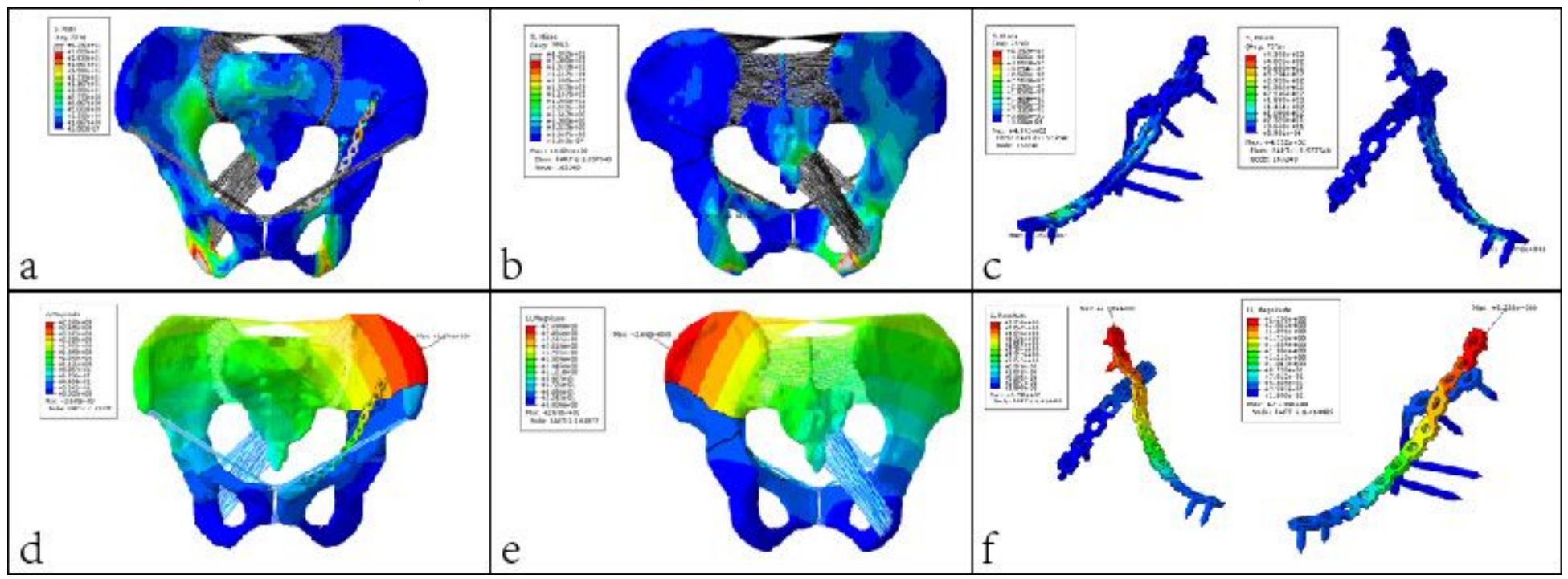

Figure 5

Nephogram of stress $(a / b / c)$ and displacement $d / e / f)$ under vertical loading in CRP 


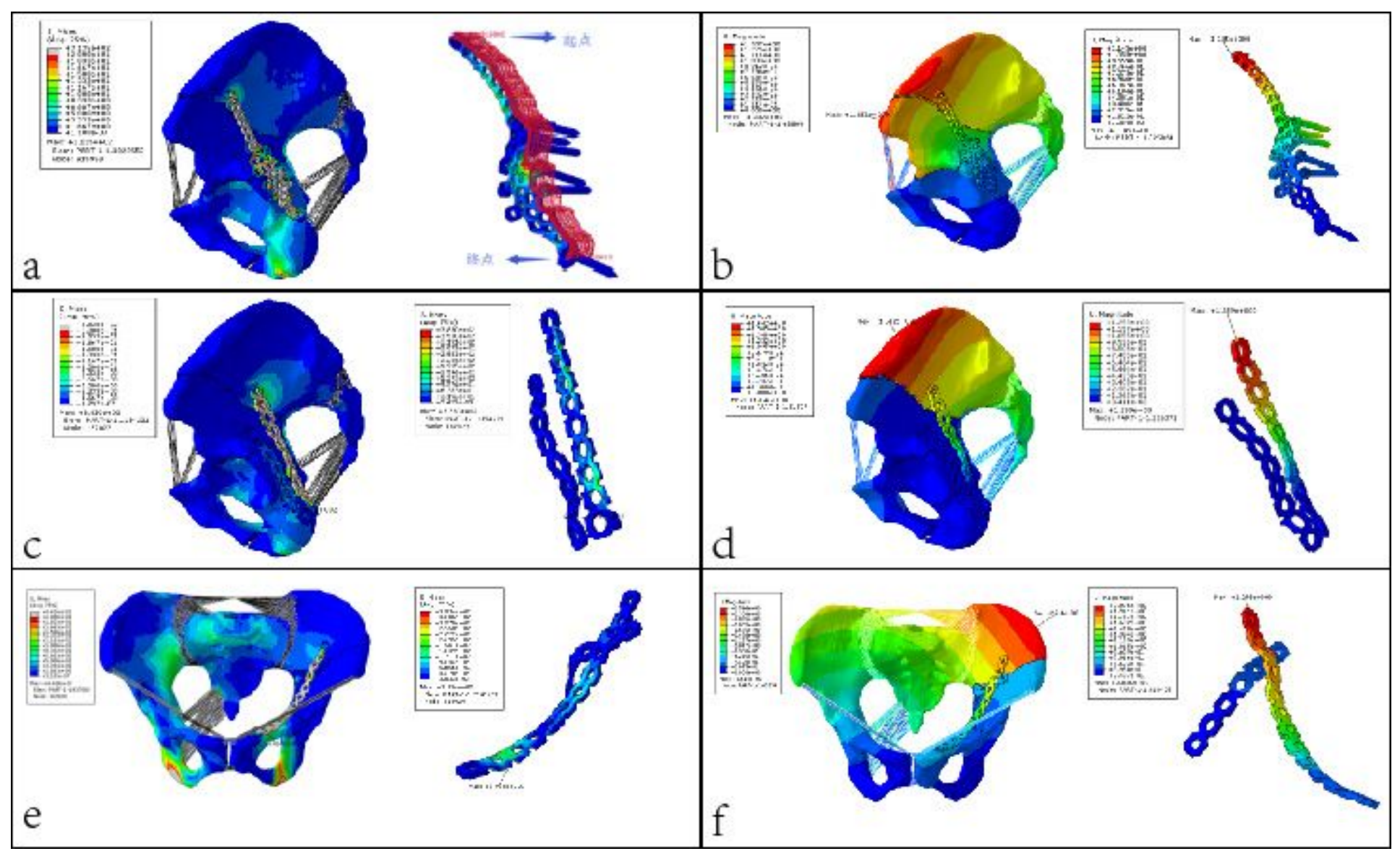

Figure 6

Stress and displacement distribution nephogram of turning right . a /b PASP, c/d DRP, e/f CRP 


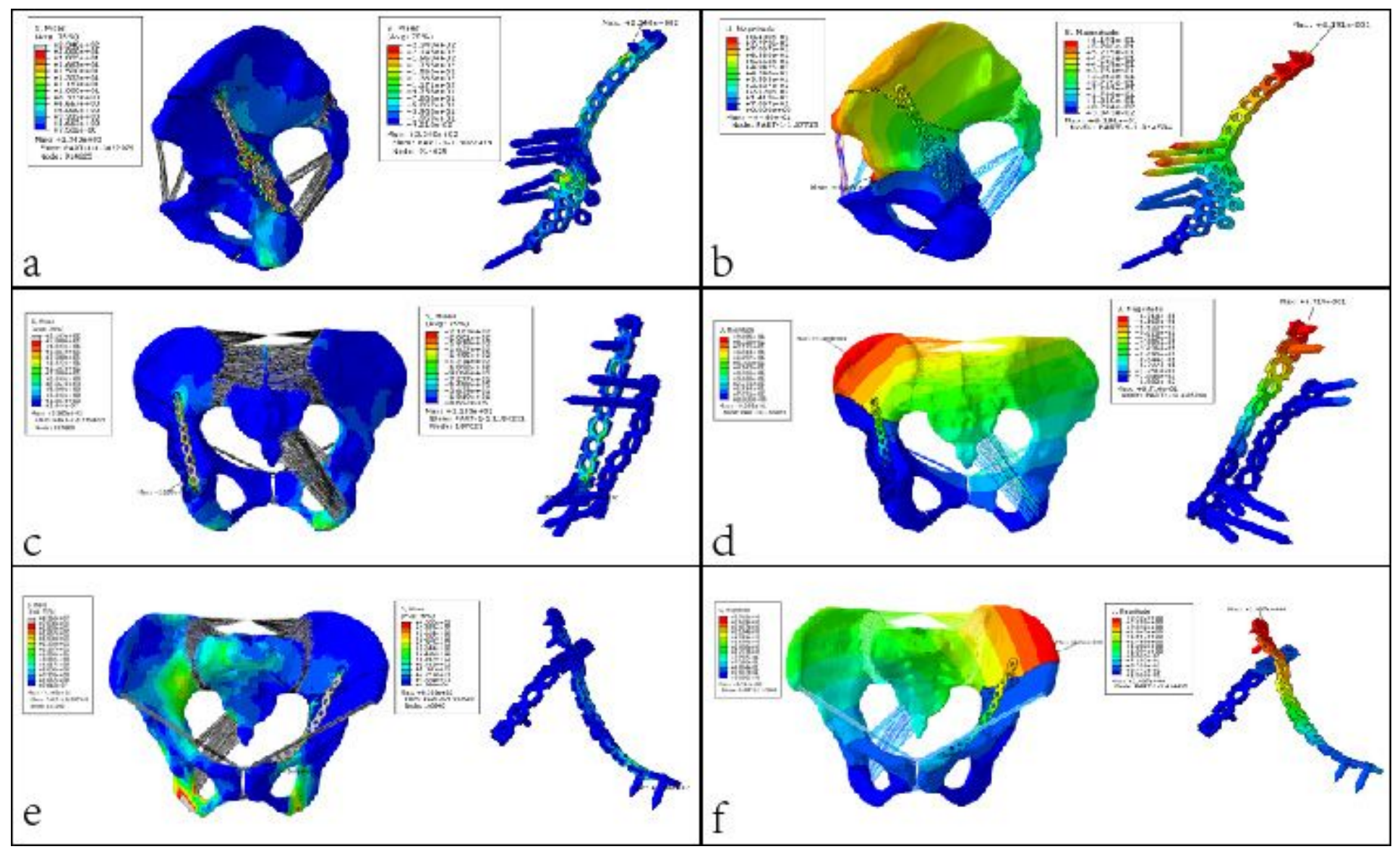

Figure 7

Stress and displacement distribution nephogram of turning left . a /b PASP, c/d DRP, e/f CRP 

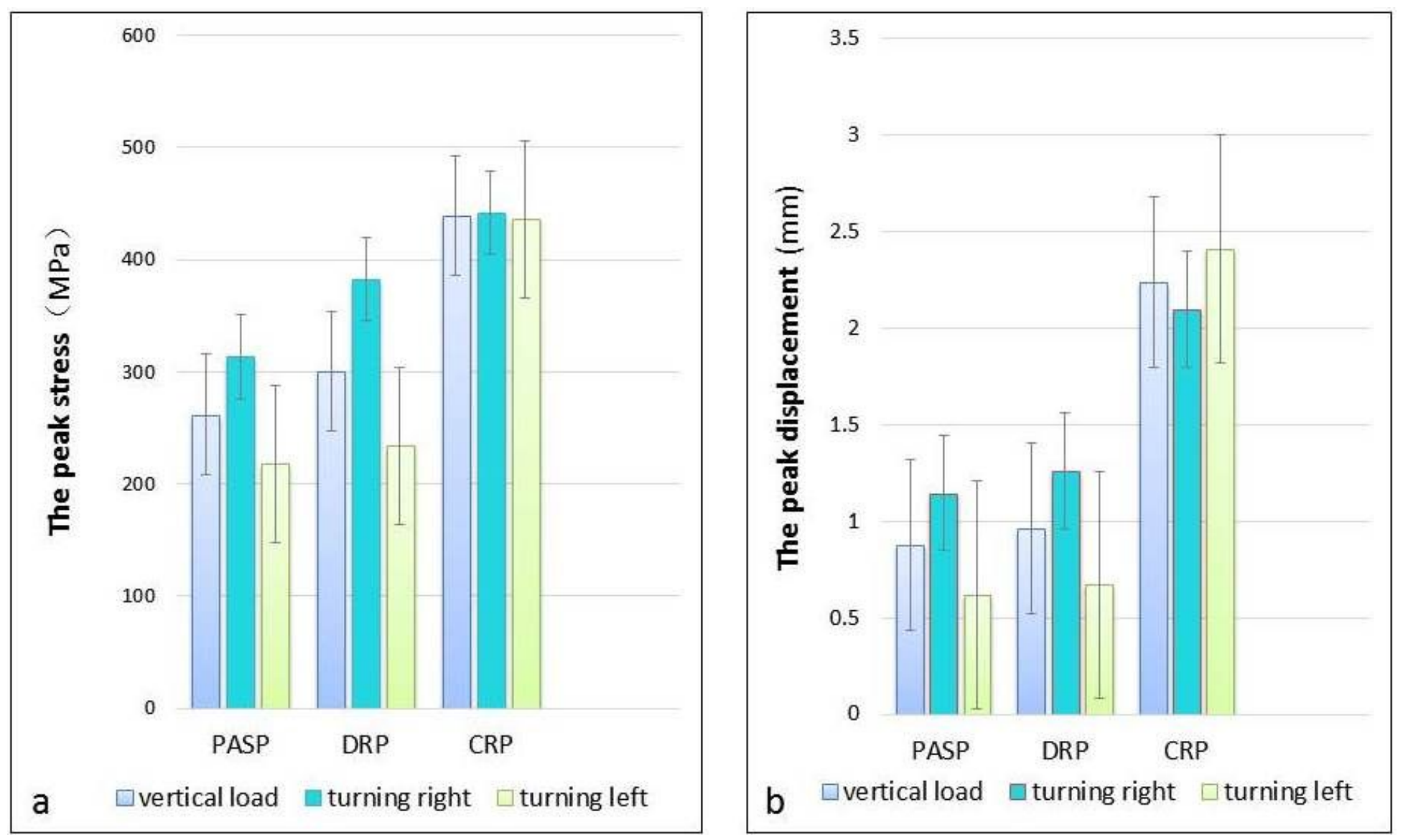

\section{Figure 8}

The peak stress ( $a$ ) and displacement b of three type s of impants for both column acetabular fractures

\section{Supplementary Files}

This is a list of supplementary files associated with this preprint. Click to download.

- Table1Materialpropertiesofthesevenpelvicligaments.pdf 